\title{
NEW APPROACH IN THE TREATMENT OF OPHTHALMIC NEOVASCULAR DISORDERS: USING FUSION PROTEIN AFLIBERCEPT
}

\author{
Kaloyan D. Georgiev', Dobrin D. Georgiev², and Dobrin S. Georgiev² \\ ${ }^{1}$ Department of Preclinical and Clinical Sciences, Sector Pharmacology and Toxicology, \\ Medical University, Varna, Bulgaria, and ${ }^{2}$ Private Eye Hospital Assoc. Prof. Georgiev, Varna, Bulgaria
}

The aim of this review is to appraise the usage of a newly approved anti-vascular endothelial growth factor (anti-VEGF) fusion protein, aflibercept, in ocular neovascular disorders such as diabetic retinopathy and age-related macular degeneration. Aflibercept is a soluble fusion protein, which combines ligand-binding elements taken from the extracellular domains of VEGF receptors 1 and 2 fused to the Fc portion of IgG. This protein contains all human amino acid sequences, which minimizes the risk for immunogenicity in human patients. In this short review we investigate the available literature and data from clinical studies on the efficacy, pharmaceutical and pharmacological properties of aflibercept, and identify its possible advantages over commercially available anti-VEGF drugs. Biomed Rev 2014; 25: 59-65

Key words: diabetic macular edema, vascular endothelial growth factor, anti-vascular endothelial growth factor drugs, aflibercept

\section{INTRODUCTION}

Diabetic macular edema (DME) is a complication of diabetic retinopathy and is a leading cause of blindness. Nowadays more people suffering from diabetes are diagnosed with DME. Diabetic macular edema, defined as retinal thickening within 2 disc diameters of the center of the macula, results from retinal microvascular changes that compromise the blood-retinal barrier, causing leakage of plasma constituents into the surrounding retina and, consequently, retinal edema (1). Anti-vascular endothelial growth factor (anti-VEGF) drugs and steroids have been introduced as potential alternatives to laser photocoagulation, the basis of treatment. Chronology of the development of the new drugs is shown on Figure 1.

Ocular angiogenesis can be physiological or pathological. The physiological ocular angiogenesis is occurring primarily during embryonic development (2). Ocular angiogenesis in adults is usually pathological and is a major cause of vision loss and blindness due to conditions as presented in Table 1.

Received 15 November 2014, revised 25 November 2014, accepted 27 November 2014

Correspondence to Dr Kaloyan D. Georgiev, Department of Preclinical and Clinical Sciences, Sector Pharmacology and Toxicology, Medical University, BG-9002 Varna, Bulgaria. Phone: +359 898343 274, E-mail: kalgeorgiev@hotmail.com 


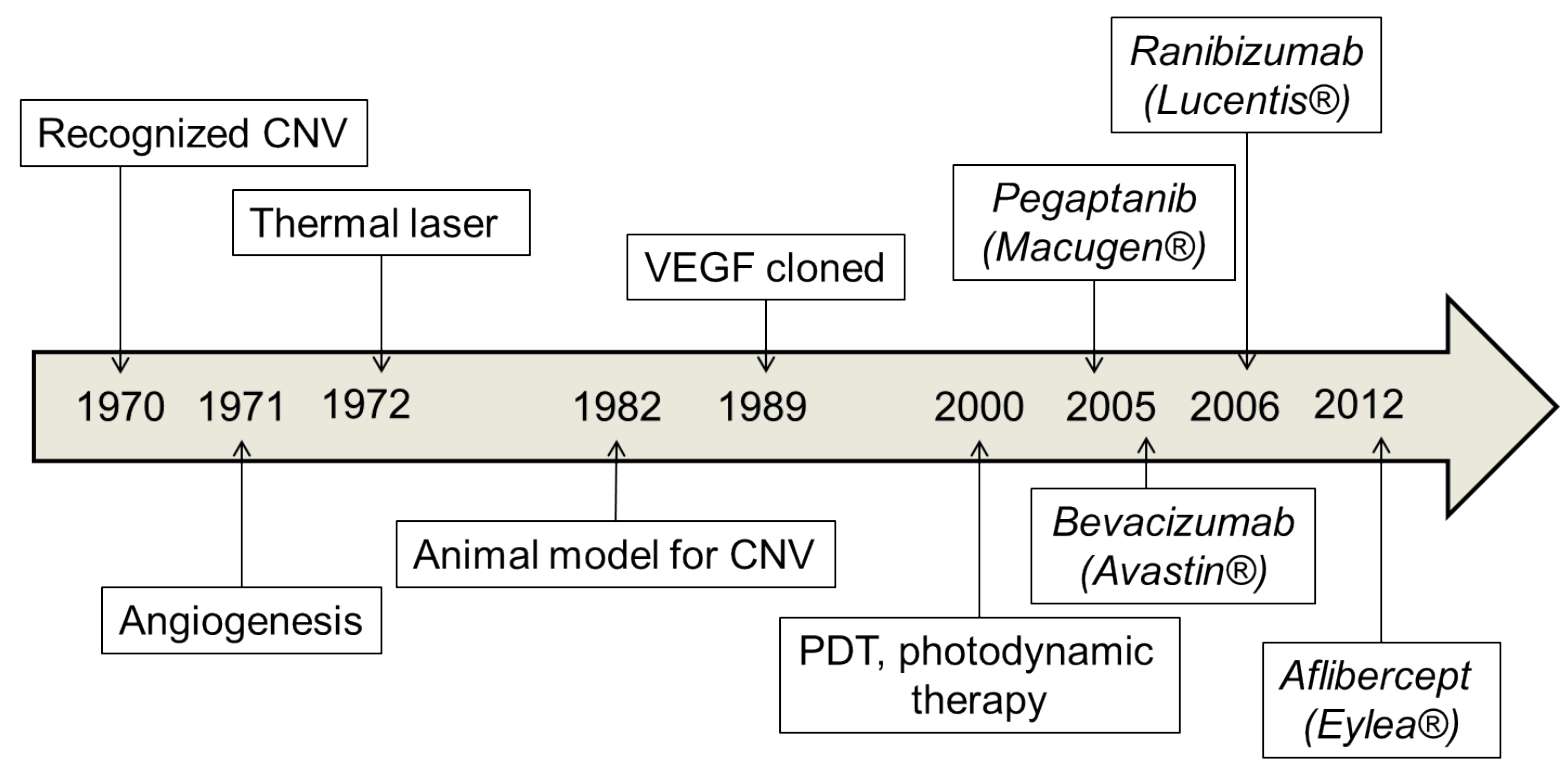

Figure 1. Timeline of discovery of therapy for neovascular age-related macular degeneration. CNV, choroidal neovascularization; VEGF, vascular endothelial growth factor; PDT, photodynamic therapy.

Table 1. Examples of ophthalmic neovascular disorders

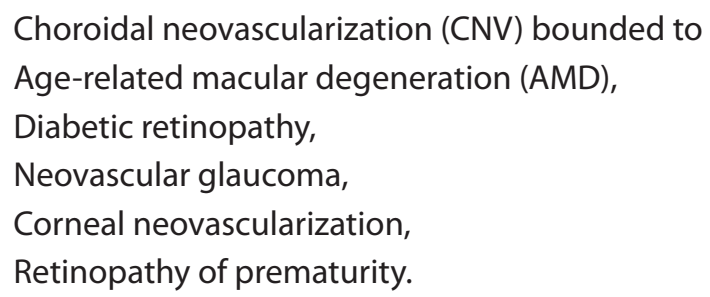

Vascular endothelial growth factor (VEGF)-A appears to be necessary for growth of blood vessels in a variety of normal and pathological circumstances (3). The VEGF family consists of five related glycoproteins, VEGF-A, VEGF-B, VEGF-C, VEGF-D, VEGF-E, and placental growth factor (PlGF) (4). VEGF has two main receptors in normal biological systems: VEGFR1 and VEGFR2. VEGFR2 mediates most of the endothelial cell proliferating activity of VEGF, whereas VEGFR1 mediates other activities of VEGF, such as a chemoattraction (5). Both receptors are important for the angiogenic-promoting properties of VEGF.

\section{VEGF TRAP-EYE MECHANISM OF ACTION}

This new approach to the treatment of wet AMD was developed to enhance the therapeutic activity - significant improvement in visual acuity and reducing frequency of administration. Aflibercept/VEGF trap-eye (VTE) is a soluble fusion protein, which combines ligand-binding elements taken from the second binding domain of the receptor VEGFR1 and the third binding domain of the receptor VEGFR2 fused to the $\mathrm{Fc}$ portion of $\operatorname{IgG}_{1}$ (Fig.2).

Each arm of VEGF Trap-Eye binds to the binding interface on each pole of the active VEGF or PIGF dimer. This forms a stable and inert 1:1 complex with the growth factor (6) uniquely binding the dimer on both sides. The Trap is aptly named, since the molecule isolates (or traps) the dimer, forming inert complexes with the growth factor (6) that do not interact with another VEGF Trap molecule. This blocks and effectively arrests the VEGF angiogenesis cascade. It also prevents the creation of multimeric complexes that can aggregate and cause immune responses in body tissues. VEGF Trap-Eye binds VEGF more tightly than native receptors, blocking cell-surface receptor activation (7). 


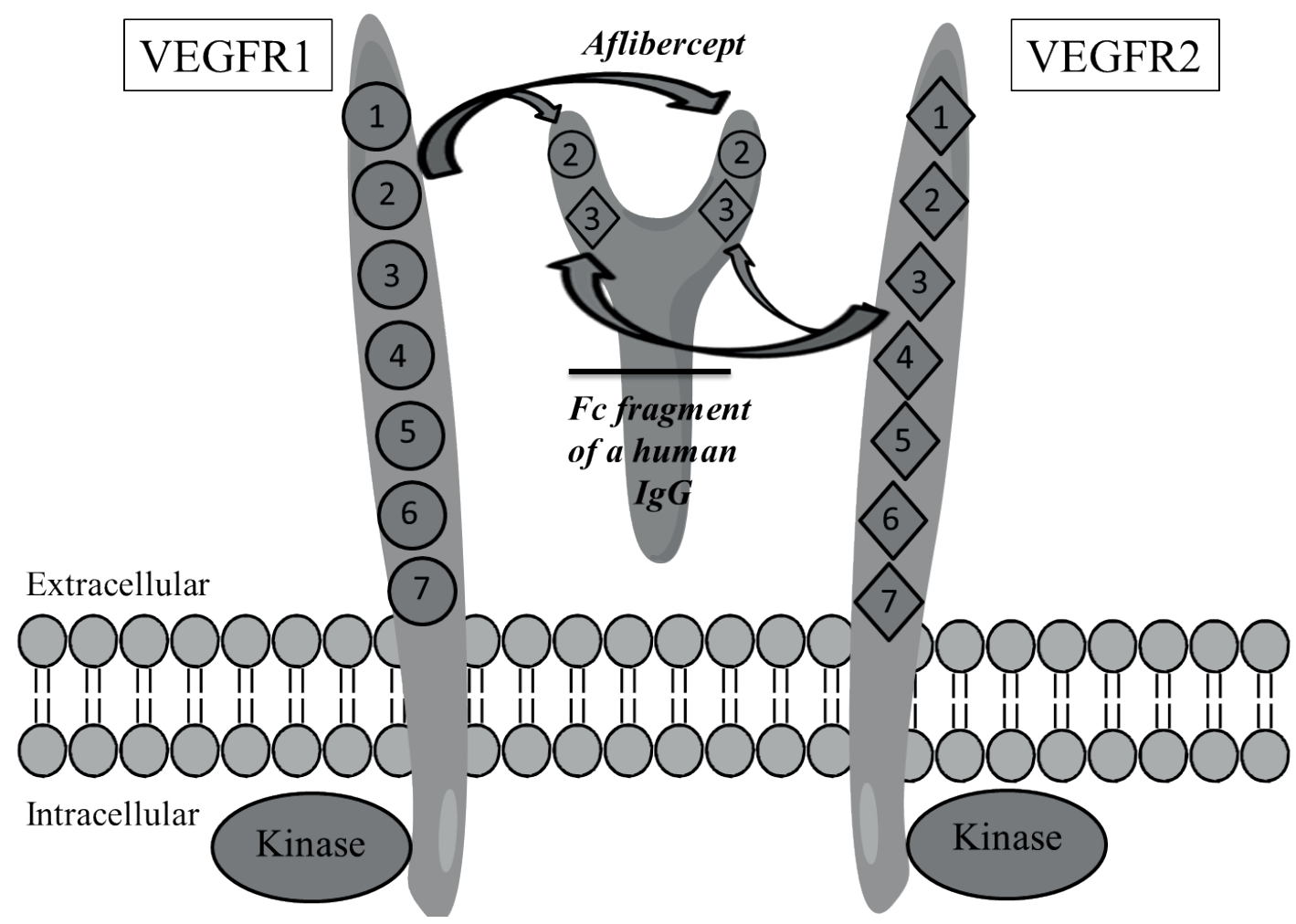

Figure 2. Creation of fusion protein - aflibercept through fusing the second VEGF-binding domain from VEGFR1 and the third VEGF-binding domain from VEGFR2 to the Fc backbone of an IgG1 molecule.

\section{INTRAOCULAR AND SYSTEMIC PHARMACOKINETIC OF AFLIBERCEPT}

Preclinical studies on intraocular pharmacokinetics are conducted on rabbits. The half-life of aflibercept in these studies was established to be 4.7 days (8). The half-life is close to that of bevacizumab (4.32 days), but is significant higher than ranibizumab (2.88 days) $(9,10)$. Intraocular pharmacokinetics, and in particular the half-life, is related to the molecular weight of the drugs. The differences in molecular size of aflibercept, ranibizumab and bevacizumab are given in Table 2 .

Table 2. Molecular weight of aflibercept, ranibizumab and bevacizumab

\begin{tabular}{|c|c|}
\hline Drug & Molecular weight \\
\hline Aflibercept & $97 \mathrm{kD}$ \\
\hline Ranibizumab & $47 \mathrm{kD}$ \\
\hline Bevacizumab & $149 \mathrm{kD}$ \\
\hline
\end{tabular}

Aflibercept is a dimeric glycoprotein with a protein molecular weight of $97 \mathrm{kD}$, but also contains some percent of glycosylation, which is leading to an additional $15 \%$ of the total molecular mass, resulting in a total molecular weight of $115 \mathrm{kD}$. That is why eye pharmacokinetic behavior of aflibercept is similar to bevacizumab. There are limited data in human eyes studies, but since the intraocular half-life of a macromolecule is primarily determined by its molecular size, we could use mathematical equation to predict it. By using such an equation, half-life of aflibercept is estimated to be 7.1 days (11). Experimental studies confirm a very good penetrationin all layers of the retina (12). After intravitreal injection of $2 \mathrm{mg}$ of aflibercept (the highest dose used in pivotal ophthalmologic trials), the maximum plasma concentration detected in 1-3 days is significantly (200-fold) lower than the concentration required for maximal systemic VEGF binding. The detection in plasma is as a free drug (a minor quantity) or in a complex bound with VEGF. The drug is rapidly cleared from circulation via pinocytotic proteolysis and glomerular 
filtration after forming a complex with VEGF via the same pathways that metabolize antibodies. The systemic half-life of the unbound aflibercept is 1.5 days, which is inferior to that of bevacizumab (20 days) and close to the systemic halflife of ranibizumab (6 hours) (11).

\section{PHARMACODYNAMIC AND DOSAGE FORMS OF AFLIBERCEPT}

The binding affinity of aflibercept to the VGFR- $\mathrm{A}_{165}$ assessed by the equilibrium dissociation constant $\left(\mathrm{K}_{\mathrm{d}}\right)$ was 0.49 pM. (14) Its affinity was 19- and 181-fold higher than the native VEGF receptors fused to Fc (VEGFR-1, $9.33 \mathrm{pM}$ or VEGFR-2, $88.8 \mathrm{pM}$, respectively). All three anti-VEGF drugs bind with high affinity to theVGFR-A ${ }_{165}$, but aflibercept have shown approximately 100 -fold lower (i.e. the binding affinity was $\sim 100$-fold tighter) than that for ranibizumab $(\mathrm{Kd}=46 \mathrm{pM})$ and bevacizumab $(\mathrm{Kd}=58 \mathrm{pM})$. (14) In addition, aflibercept possesses a high binding affinityfor PlGF-2 $(\mathrm{Kd} \sim 45 \mathrm{pM})(15)$.

Nowadays, aflibercept has ophthalmologic and oncologic use registration. Dosage forms that are used in ophthalmology are preservative-free, sterile, aqueous solution in a singleuse, glass vial designed to deliver $0.05 \mathrm{~mL}$ (50 microliters) of aflibercept $(40 \mathrm{mg} / \mathrm{mL}$ in $10 \mathrm{mM}$ sodium phosphate, $40 \mathrm{mM}$ sodium chloride, $0.03 \%$ polysorbate 20 , and $5 \%$ sucrose, $\mathrm{pH}$ 6.2). The trade name, under which the product is registered, is Eylea ${ }^{\circledR}$ (Regeneron Pharmaceuticals, Tarrytown, NY, USA and Bayer, Berlin, Germany). In oncology practice the product is registered under another generic name - ziv-aflibercept, with trade name Zaltrap ${ }^{\circledR}$ (Sanofi-Aventis, Paris, France and Regeneron Pharmaceuticals, Tarrytown, NY, USA). It was approved for the treatment of patients with metastatic colorectal cancer who had been previously treated (16). According to the manufacturer, this drug must not be administered intravitreally due to its hyperosmolarity (1000 mOsm). The dosage that is used in ophthalmology is 100 -fold lower than the dose allowed in oncology (4-6 mg/kg) (17).

\section{THERAPEUTIC EFFICACY}

Aflibercept is currently being approved for the treatment of two eye diseases: neovascular (wet) age-related macular degeneration (AMD) and macular edema following central retinal vein occlusion (CRVO).

\section{Neovascular age-related macular degeneration}

In the first clinical experience, the purpose has been to evaluate the maximum tolerated dose, the bioactivity, the safety and tolerability of intravitreally administered aflibercept. This was done in Phase I study named Clinical Evaluation of Antiangiogenesis in the Retina Intravitreal Trial (CLEAR-IT 1) (18). The dosage range of aflibercept was between $0,05-4$ $\mathrm{mg}$, which were tested on 21 patients. At 6 weeks after a single injection, most patients experienced an improvement in visual acuity (mean visual gain, 4.4 letters) and showed a decrease in macular thickness $(-105 \mu \mathrm{m})$. Figure 3 shows the result obtained with our patient suffering from neovascular (wet) AMD: after treatment with aflibercept there was an almost $50 \%$ reduction in macular thickness and almost the same percent improvement in visual acuity (unpublished data).

Aflibercept is well-tolerated with no visible ocular inflammation seen. In the second clinical phase (CLEAR-IT 2), the purpose has been to evaluate dose and dosing range interval, the patients $(n=159)$ being randomized into five treatment groups: the first two groups received 3 aflibercept injections of $0.5 \mathrm{mg}$ or $2 \mathrm{mg}$ monthly and the other three groups received only one aflibercept injection of $0.5 \mathrm{mg}, 2 \mathrm{mg}$, or 4 mg monthly (19). The results of this clinical stage can be summarized as follows: (i) the reduction in macular thickness experienced by the patients receiving three monthly injections exceeded that of patients treated only once (20), (ii) patients initially treated with 2 mg every 4 weeks had the best visual improvement (mean gain of 9 letters) and also aflibercept can be applied as needed with excellent gains in vision (21). Thus, three different dosing schedules were identified for the clinical phase III studies: $0.5 \mathrm{mg}$ monthly (0.5Q4), 2 mg monthly (2Q4), or $2 \mathrm{mg}$ every 2 months (2Q8) after the loading phase of three initial monthly doses and all of them compared to the standard dosing schedule for ranibizumab $0.5 \mathrm{mg}$ monthly. The studies VEGF Trap-eye Investigation of Efficacy and Safety in Wet AMD (VIEW) 1 and 2, have been conducted to evaluate efficacy of aflibercept to standard treatment - ranibizumab, against which all subsequent drugs should be compared. The View 1 study enrolled 1217 patients in the US and Canada, and the View 2 study enrolled 1240 patients in Europe, Asia, Japan, and Latin America.In both studies, the primary efficacy endpoint has been the proportion of patients who maintained vision, defined as losing fewer than 15 letters of visual acuity at week 52 compared to baseline. Data are available through week 52 . Both aflibercept $2 \mathrm{Q} 8$ and aflibercept $2 \mathrm{Q} 4$ groups were shown to have efficacy that was clinically equivalent to the ranibizumab 0.5 mg Q4 group.In VIEW 1, patients receiving $2 \mathrm{mg}$ of aflibercept every 4 weeks gained more vision than those receiving ranibizumab $(+10.9$ letters versus +8.1 letters; $p=0.0054)$ (22). Improvements in macular thickness were not statisti- 

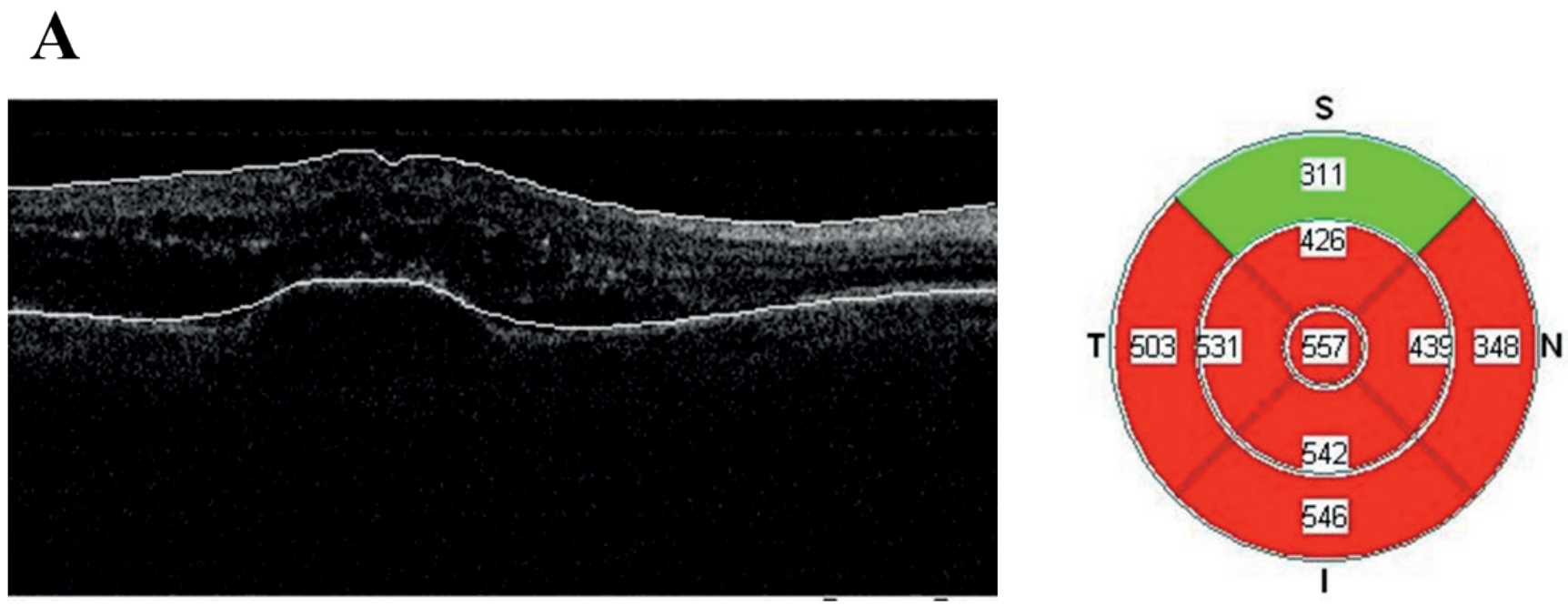

\section{B}
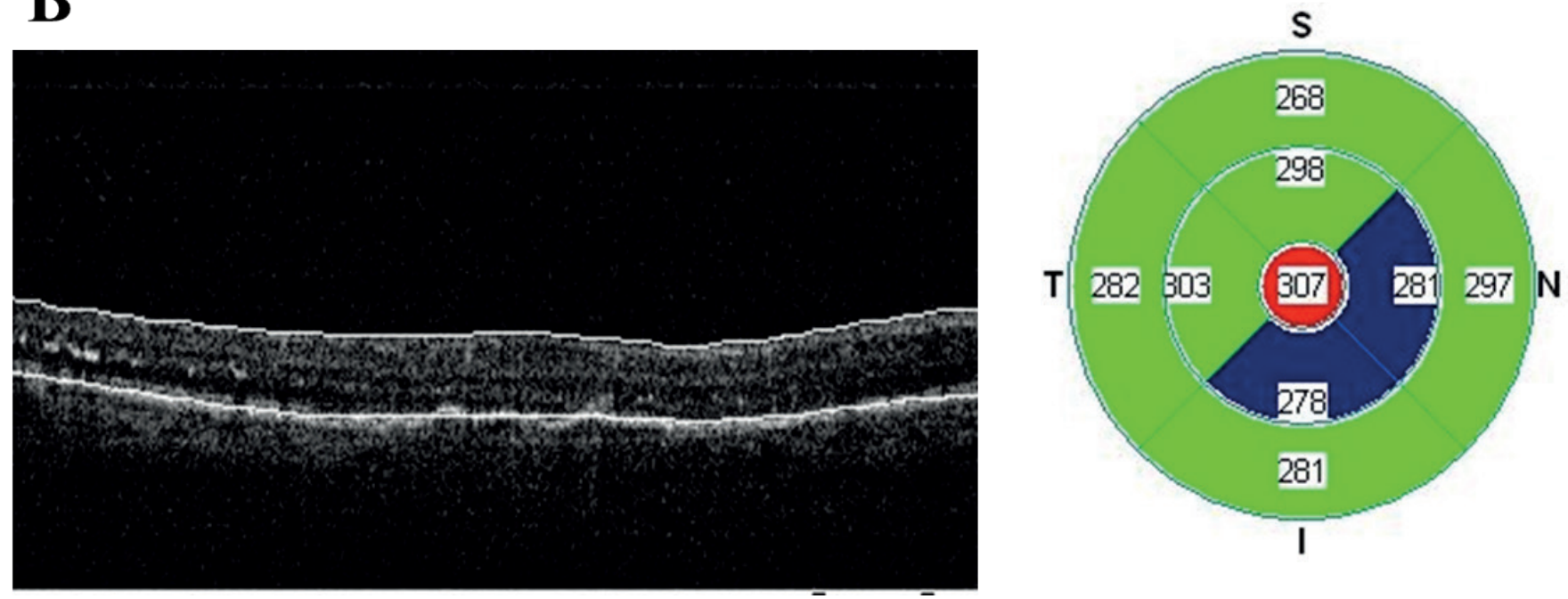

Figure 3.A Macular thickness before administration of aflibercept-557,90 $\mu \mathrm{m}$; B Macular thickness four weeks after administration of aflibercept $-307,00 \mu \mathrm{m}$;

cally different in the treatment groups. VIEW 2 patients receiving $2 \mathrm{mg}$ of aflibercept every 8 weeks showed bimonthly fluctuations in macular thickness without corresponding fluctuations in visual acuity (22). The safety of aflibercept was excellent and was comparable with that of ranibizumab in both the VIEW 1 and VIEW 2 studies. Severe extraocular adverse events such as stroke and myocardial infarction occurred with similar frequencies in patients receiving aflibercept $(0.7 \%$ and $2.6 \%$, respectively) and in patients receiving ranibizumab (1.6\% and $2.6 \%$, respectively) in both VIEW trials. Both studies showed that all 3 regimens of aflibercept were noninferior to the ranibizumab monthly regimen and the advantages of aflibercept's regimen are the fewer injections (no loss of efficacy) and less risk of endophthalmitis.

\section{Macular edema following central retinal vein occlusion (CRVO)}

Therapeutic activity of aflibercept in the treatment of macular edema following CRVO was proved in two phase III clinical studies - COPERNICUS (Controlled Phase 3 Evaluation of Repeated intravitreal administration of VEGF Trap-Eye In Central retinal vein occlusion: Utility and Safety) (23) and 
GALILEO (General Assessment Limiting Infiltration of Exudates in central retinal vein Occlusion with EYLEA) (24). The first six months of the both studies were identical, patients were randomized into two groups - receiving intravitreal aflibercept $2 \mathrm{mg}$ monthly and those who received intravitreal placebomonthly.In the second six months, GALILEO study was design to continue treatment group with aflibercept on the PRN (provided as needed) basis and the control group with placebo (24), while in the COPERNICUS study (23), all patients were treated with aflibercept on a PRN basis.In both the COPERNICUS and GALILEO studies, aflibercept injection resulted in an improvement in visual acuity of .15 letters in $56.1 \%$ and $60.2 \%$ of patients, respectively, at week 24 compared with those receiving sham injections $(12.3 \%$ and $22.1 \%$, respectively). At week 52 in the COPERNICUS study, the improvement in visual acuity was $55.3 \%$ in the aflibercept/aflibercept PRN patients compared with $30.1 \%$ in the placebo/aflibercept PRN patients. In the GALILEO study, in which control patients did not receive any aflibercept injections, the improvement was $60.2 \%$ and $32.4 \%$, respectively. The results of these studies showed that it is possible to maintain an excellent visual outcome and to extend the range of administration while using the PRN strategy. These data indicate that aflibercept provides benefits to patients with CRVO and using this drug as needed may become a first line approach that will reduce the burden of monthly injections.

\section{CONCLUSION}

It can be stated that aflibercept represents an attractive alternative to the available anti-VEGF drugs as it leads to similar improvements in visual outcomes and has a longer duration of action, due to stronger and prolonged binding to the VEGF-A receptor, which allows a decrease of the frequency of injections.

\section{CONFLICT OF INTEREST}

The authors declare no conflict of interest.

\section{REFERENCES}

1. Albert DM, Jakobiec FA. Principles and Practice of Ophthalmology. 2nd edition, Philadelphia: WB Saunders Co; 2000.

2. Gariano RF, Gardner TW.Retinal angiogenesis in development and disease. Nature 2005;438:960-966.

3. Coultas L, Chawengsaksophak K, Rossant J. Endothelial cells and VEGF in vascular development. Nature 2005;438: 937-945.
4. Ferrara N, Gerber HP, LeCouter J. The biology of VEGF and its receptors. Nat Med 2003;9:669-676.

5. Olsson AK, Dimberg A, Kreuger J, Claesson-Welsh L. VEGF receptor signalling-in control of vascular function. Nat Rev Mol Cell Biol 2006;7:359-371.

6. Rudge JS, Holash J, Hylton D, et al. Inaugural Article: VEGF Trap complex formation measures production rates of VEGF, providing a biomarker for predicting efficacious angiogenic blockade. ProcNatlAcadSci USA 2007;104:18363-18370.

7. Rudge JS, Thurston G, Davis S, et al. VEGF trap as a novel antiangiogenic treatment currently in clinical trials for cancer and eye diseases, and VelociGene-based discovery of the next generation of angiogenesis targets. Cold Spring Harbor Symp Quant Biol 2005;70:411-418.

8. Furfine E, Coppi A, Koehler-Stec E, Zimmer E, Tu W, Struble C. Pharmacokinetics and ocular tissue penetration of VEGF Trap after intravitreal injections in rabbits. Invest Ophthalmol Vis Sci 2006; 47, E-abstract 1430.

9. Bakri SJ, Snyder MR, Reid JM, Pulido JS,Ezzat MK, Singh RJ. Pharmacokinetics of intravitreal ranibizumab (Lucentis).Ophthalmology 2007; 114: 2179-2182.

10. Bakri SJ, Snyder MR, Reid JM, Pulido JS,Singh RJ. Pharmacokinetics of intravitreal bevacizumab (Avastin). Ophthalmology 2007; 114, 855-859.

11. Stewart MW. What are the half-lives ofranibizumab and aflibercept (VEGFTrap-Eye) in human eyes? Calculations with a mathematical model. Eye Rep 2011; 1:e5.

12. Pieramici DJ, Rabena MD. Anti-VEGF therapy: Comparison of current and future agents. Eye (Lond) 2008;22:1330-1336. DOI: 10.1038/eye.2008.88.

13. EYLEA ${ }^{\mathrm{TM}}$ (aflibercept) injection: US prescribing information. Tarrytown, NY, USA; Regeneron Pharmaceuticals, Inc. Available from: http://www.regeneron.com/ Eylea/eylea-fpi.pdf. Accessed April 2, 2012.

14. Papadopoulos N, Martin J, Ruan Q, et al. Binding and neutralization of vascularendothelial growth factor (VEGF) and related ligands by VEGF Trap, ranibizumaband bevacizumab. Angiogenesis 2012; 15:171-185. DOI: 10.1007/s10456-011-9249-6.

15. Holash J, Davis S, Papadopoulos N, et al. VEGF-Trap: a VEGF blocker with potent antitumor effects. ProcNatlAcadSci USA2002; 99:11393-11398.

16. Gaya A, Tse V. A preclinical and clinical review of aflibercept for the management of cancer. Cancer Treat Rev 2012;38:484-493. DOI: 10.1016/j.ctrv.2011.12.008.

17. Mitchell EP. Targeted therapy for metastatic colorec- 
tal cancer: Role of aflibercept. Clin Colorectal Cancer 2013; 12: 73-85. DOI: 10.1016/j.clcc.2012.08.001.

18. Nguyen QD, Shah SM, Hafiz G, et al. CLEAR-AMD 1 Study Group. A phase I trial of an IV-administered vascular endothelial growth factor trap for treatment in patients with choroidal neovascularization due to age-related macular degeneration. Ophthalmology 2006;113:1522.e1-1532.e14.

19. Heier JS, Boyer D, Nguyen QD, et al. CLEAR-IT 2 Investigators. The 1-year results of CLEAR-IT 2, a phase 2 study of vascular endothelial growth factor trap-eye dosed as-needed after 12-week fixed dosing. Ophthalmology 2011;118:1098-1106. DOI: 10.1016/j.ophtha.2011.03.020.

20. Brown DM, Heier JS, Ciulla T, et al. CLEAR-IT 2 Investigators. Primary endpoint results of a phase II study of vascular endothelial growth factor trap-eye in wet agerelated macular degeneration. Ophthalmology 2011;118: 1089-1097. DOI: 10.1016/j.ophtha.2011.02.039.

21. Stewart MW, Rosenfeld PJ, Penha FM, et al. Pharma- cokinetic rationale for dosing every 2 weeks versus 4 weeks with intravitreal ranibizumab, bevacizumab, and aflibercept (vascular endothelial growth factor trap-eye). Retina 2012;32:434-457. DOI: 10.1097/ IAE.0B013E31822C290F.

22. Heier JS, Brown DM, Chong V, et al. VIEW 1 and VIEW 2 Study Groups. Intravitreal aflibercept (VEGF trap-eye) in wet age-related macular degeneration. Ophthalmology 2012;119:2537-2548. DOI: 10.1016/j.ophtha.2012.09.006.

23. Brown DM, Heier JS, Clark LW, et al. Intravitreal aflibercept injection for macular edema secondary to central vein occlusion: 1-year results from the phase 3 COPERNICUS study. Am J Ophthalmol 2013;155: 429437. DOI: 10.1016/j.ajo.2012.09.026.

24. Holz FG, Roider J, Ogura Y, et al. VEGF Trap-Eye for macular oedema secondary to central retinal vein occlusion: 6-month results of the phase III GALILEO study. Br J Ophthalmol 2013;97:278-284. DOI: 10.1136/ bjophthalmol-2012-301504. 